\title{
On CR-submanifolds of $(\varepsilon)$-paracontact Sasakian manifold
}

\author{
Barnali Laha ${ }^{1}$ and Arindam Bhattacharyya ${ }^{2}$ \\ ${ }^{1}$ Department of Mathematics, Jadavpur University, Kolkata, India \\ 2 Department of Mathematics, Jadavpur University, Kolkata, India
}

\begin{abstract}
The object of this paper is to prove some of the properties of $(\varepsilon)$-paracontact Sasakian manifold. This paper is summarized as follows: Section (1) is introductory. In Section (2) we sketch the notion of $(\varepsilon)$ paracontact metric manifold and $(\varepsilon)$-paracontact Sasakian manifold. Section (3) and (4) deals with some properties like $D$-totally, $D^{\perp}$-totally, mixed totally geodesic etc. CR-submanifold of an $(\varepsilon)$-paracontact Sasakian manifold. Section (5) and (6) gives some idea of geometry of leaves and contact CR-product. Finally we have given an example of $(\varepsilon)$-paracontact Sasakian manifold.
\end{abstract}

Key Words and Phrases: $(\varepsilon)$-paracontact Sasakian manifold, $D\left(D^{\perp}\right)$-totally geodesic, mixed totally geodesic, contact CR-product.

\section{Introduction}

An almost paracontact structure $(\phi, \xi, \eta)$ satisfying $\phi^{2}=I-\eta \otimes \xi$ and $\eta(\xi)=1$ on a differentiable manifold was introduced by Sato [7] in 1976. This structure is analogous to almost contact structure ([6], [14]) and is very much related to almost product structure (in contrast to almost contact structure which is related to almost complex structure ). An almost contact manifold is always odd dimensional whereas an almost paracontact manifold can be even dimensional also.

Takahashi[16] in 1969, gave the notion of almost contact manifold equipped with an associated pseudo-Riemannian metric. Actually, he studied Sasakian manifolds endowed with an associated pseudo-Riemannian metric. These indefinite almost contact metric manifold and indefinite Sasakian manifolds are also known as $(\varepsilon)$ - almost contact metric manifolds and $(\varepsilon)$ - Sasakian manifolds, respectively ([3], [9], [10]). Again, Matsumoto ([12]) in 1989, replaced the structure vector field $\xi$ by $-\xi$ in an almost paracontact manifold and have also associated a Lorentzian metric with the resulting structure and defined it as Lorentzian almost paracontact manifold. The semi-Riemannian metric has index 1 and the structure vector field $\xi$ is lightlike in a Lorentzian almost paracontact manifold, which was introduced by Matsumoto ([12]). These circumstances has motivated M.M.Tripathi et.al.([15]) to draw a relation between a semi-Riemannian metric ( not necessarily Lorentzian ) and an almost paracontact structure, and named this indefinite almost paracontact metric structure as an $(\varepsilon)$ - almost paracontact structure, where the structure vector field $\xi$ will be spacelike or timelike according as $\varepsilon=1$ or $\varepsilon=-1$. Authors have discussed $(\varepsilon)$ - almost paracontact manifolds and in particular $(\varepsilon)$ - Sasakian manifolds in([15]).

\section{Preliminaries}

Definition 1 Let $\tilde{M}$ be an $n$-dimensional almost paracontact manifold [7] endowed with an almost paracontact structure $(\phi, \xi, \eta)$ consisting of a tensor field $\phi$ of type $(1,1)$, a structure vector field $\xi$ and 1 form $\eta$ satisfying:

$$
\phi^{2}=I-\eta \otimes \xi, \eta(\xi)=1, \phi(\xi)=0, \eta \circ \phi=0 .
$$

In this paper we have assumed $X, Y, Z \in \chi(M)$ where $\chi(M)$ is the Lie Algebra of vector field in $\tilde{M}$, unless specifically stated otherwise. A semi-Riemannian metric [5] on a manifold $\tilde{M}$, is a nondegenerate symmetric tensor field $g$ of type $(0,2)$. If this metric is of index 1 then it is called Lorentzian 
metric ([8]). Let $g$ be semi-Riemannian metric with index 1 in an $n$-dimensional almost paracontact manifold $\tilde{M}$ such that,

$$
\tilde{g}(\phi X, \phi Y)=\tilde{g}(X, Y)-\varepsilon \eta(X) \eta(Y),
$$

where $\varepsilon=+1$ or -1 . Then $\tilde{M}$ is called an almost paracontact metric manifold associated with an $\varepsilon$ -almost paracontact metric structure $(\phi, \xi, \eta, g, \varepsilon)([15])$. In case, if index $(g)=1$ then an $\varepsilon$-almost paracontact metric manifold is defined as a Lorentzian paracontact manifold and if the metric is positive definite, then an $\varepsilon$-almost paracontact metric manifold is the usual almost paracontact metric manifold [7].

The condition (2) is equivalent to

equipped with

$$
g(X, \phi Y)=g(\phi X, Y)
$$

$$
g(X, \xi)=\varepsilon \eta(X) .
$$

From (4), it is easy to see that

$$
g(\xi, \xi)=\varepsilon,
$$

i.e. structure vector field $\xi$ is never lightlike. We define

$$
\Phi(X, Y)=g(X, \phi Y),
$$

and we see that $\Phi(X, \xi)=0$.

From (6), we can also obtain

$$
\left(\nabla_{X} \Phi\right)(Y, Z)=g\left(\left(\nabla_{X} \phi\right) Y, Z\right)=\left(\nabla_{X} \Phi\right)(Z, Y) .
$$

An $(\varepsilon)$-almost paracontact metric manifold $\tilde{M}$ satisfying

$$
2 \Phi(X, Y)=\left(\nabla_{X} \eta\right)(Y)+\left(\nabla_{Y} \eta\right)(X),
$$

$\forall X, Y \in T M$, is called an $(\varepsilon)$-paracontact metric manifold ([15]).

An $(\varepsilon)$-almost paracontact metric structure is called an $(\varepsilon)$-para Sasakian structure if the following relation holds.

$$
\left(\tilde{\nabla}_{X} \phi\right)(Y)=-g(\phi X, \phi Y) \xi-\varepsilon \eta(Y) \phi^{2} X,
$$

where $\tilde{\nabla}$ is the Levi-Civita connection with respect to $g$ on $\tilde{M}$. A manifold equipped with an $(\varepsilon)$ para Sasakian structure is called an $(\varepsilon)$-para Sasakian manifold.

- $\varepsilon=1, g$ is Riemannian, then $\tilde{M}$ is usual para Sasakian manifold [7].

- $\varepsilon=-1, g$ is Lorentzian and $\xi$ is replaced by $-\xi$, then $\tilde{M}$ is called Lorentzian para Sasakian manifold [12].

$$
\text { Also we know }
$$

$$
\tilde{\nabla}_{X} \xi=\varepsilon \phi X
$$

From the definition of contact CR-submanifolds of an $(\varepsilon)$-paracontact Sasakian manifold we have, 
Definition 2 An $n$-dimensional Riemannian submanifold $M$ of an ( $\varepsilon$ )-paracontact Sasakian manifold $\tilde{M}$ is called a contact CR-submanifold if $\xi$ is tangent to $M$ and there exists on $M$ a differentiable distribution $D: x \rightarrow D_{x} \subset T_{x}(M)$, such that $(i) D_{x}$ is invariant under $\phi$; i.e., $\phi D_{x} \subset D_{x}$, for each $x \in M$ and

(ii) the orthogonal complementary distribution $D^{\perp}: x \rightarrow D_{x}^{\perp} \subset T_{x}^{\perp}(M)$ of the distribution $D$ on $M$ is totally real; i.e., $\phi D_{x}^{\perp} \subset T_{x}^{\perp}(M)$, where $T_{x}(M)$ and $T_{x}^{\perp}(M)$ are the tangent space and the normal space of $M$ at $x$.

$D\left(\right.$ resp. $\left.D^{\perp}\right)$ is the horizontal (resp. vertical) distribution. The contact CR-submanifold of an $(\varepsilon)$ paracontact Sasakian manifold is called $\xi$-horizontal (resp. $\xi$-vertical) if $\xi_{x} \in D_{x}$ (resp. $\xi_{x} \in D_{x}^{\perp}$ ) for each $x \in M$ by [11].

Let $T M$ and $T^{\perp} M$ be the Lie algebras of vector fields tangential to $M$ and normal to $M$ respectively. $h$ and $A$ denote the second fundamental form and the shape operator of the immersion of $M$ into $\tilde{M}$ respectively. If $\nabla$ is the induced connection on $M$, the Gauss and Weingarten formulae of $M$ into $\tilde{M}$ are then given respectively by

$$
\begin{aligned}
& \tilde{\nabla}_{X} Y=\nabla_{X} Y+h(X, Y), \\
& \tilde{\nabla}_{X} N=-A_{N} X+\nabla_{X}^{\perp} N,
\end{aligned}
$$

for any $X, Y$ in $T M$ and $N$ in $T^{\perp} M . \nabla^{\perp}$ is the connection on normal bundle and $A_{N}$ is the Weingarten endomorphism associated with $N$. It can be easily seen that

$$
g\left(A_{N} X, Y\right)=g(h(X, Y), N) .
$$

Now we can express a vector field $X \in T M$ as

$$
X=P X+Q X,
$$

where $P$ and $Q$ are projectors of $D$ and $D^{\perp}$ respectively and i.e. $P X$ and $Q X$ belongs to the distribution $D$ and $D^{\perp}$.

Similarly for $N \in T^{\perp} M$ we can write

$$
\phi N=B N+C N,
$$

where $B N$ (resp. $C N$ ) is vertical (resp. normal) component of $\phi N$. If we denote the orthogonal components of $\phi D$ in $T^{\perp} M$ by $\mu$ then we can write

$$
T^{\perp}=\phi D^{\perp} \oplus \mu \text { i.e. } \phi \mu=\mu .
$$

The covariant derivative of the tensor fields $\phi, P, Q$ are defined as

$$
\begin{aligned}
& \left(\tilde{\nabla}_{X} \phi\right) Y=\tilde{\nabla}_{X} \phi Y-\phi \tilde{\nabla}_{X} Y, \forall X, Y \in T \tilde{M} . \\
& \left(\tilde{\nabla}_{X} P\right) Y=\nabla_{X} P Y-P \nabla_{X} Y, \forall X, Y \in T M . \\
& \left(\tilde{\nabla}_{X} Q\right) Y=\nabla_{X}^{\perp} Q Y-Q \nabla_{X} Y, \forall X, Y \in T M .
\end{aligned}
$$



components

Using (9) and after some brief calculations we obtain on comparing the horizontal, vertical and normal

$$
\begin{aligned}
& P \nabla_{X} \phi P Y-P A_{\phi Q Y} X=\phi P \nabla_{X} Y-g(\phi X, \phi Y) P \xi-\varepsilon \eta(Y) \phi^{2} P X, \\
& Q \nabla_{X} \phi P Y-Q A_{\phi Q Y} X=B h(X, Y)-g(\phi X, \phi Y) Q \xi-\varepsilon \eta(Y) \phi^{2} Q X, \\
& h(X, \phi P Y)+\nabla_{X}^{\perp} \phi Q Y=\phi Q \nabla_{X} Y+C h(X, Y) .
\end{aligned}
$$

From (10) we get

$$
\nabla_{X} \xi+h(X, \xi)=\alpha \phi Q Y+\alpha \phi P X .
$$

Comparing L.H.S and R.H.S of (22) we get

$$
\begin{aligned}
& \nabla_{X} \xi=c \phi P X, \\
& h(X, \xi)=s \phi Q X .
\end{aligned}
$$

Also we have

$$
\nabla_{X} \xi=0
$$

if $X \in D^{\perp}$, and

$$
h(X, \xi)=0
$$

if $X \in D$.

Again,

$$
h(\xi, \xi)=0,
$$

and

$$
A_{N} \xi \in D^{\perp}
$$

III. $\quad D(D$-perp $)$-totally geodesic and $D(D$-perp $)$-umbilic contact $\mathbf{C R}$-submanifolds of an (epsilon)-paracontact Sasakian manifold.

In this section we first define $D$-totally geodesic( resp. $D^{\perp}$ - totally geodesic) and then $D$ umbilic(resp. $D^{\perp}$ - umbilic). And also prove some properties regarding these.

Definition 3 A contact CR-submanifold $M$ of an $(\varepsilon)$-paracontact Sasakian manifold $\tilde{M}$ is called $D$-totally geodesic (resp. $\quad D^{\perp}$-totally geodesic) if $h(X, Y)=0, \forall X, Y \in D \quad$ (resp. $X, Y \in D^{\perp}$ ) [1][2].

From the definition (3), we can easily conclude the following propositions.

Proposition 4 Let $M$ be a contact CR-submanifold of an $(\varepsilon)$-paracontact Sasakian manifold. Then $M$ is $D$-totally geodesic if and only if $A_{N} X \in D^{\perp}$ for each $X \in D$, where $N$ is normal vector field to $M$.

Proof. From (13) we know

$g(h(X, Y), N)=g\left(A_{N} X, Y\right)$.

If $M$ be $D$-totally geodesic we obtain 
$g(h(X, Y), N)=0$.

Therefore $g\left(A_{N} X, Y\right)=0$, and so,

$A_{N} X \in D^{\perp} \quad \forall X, Y \in D$.

Conversely, suppose that $A_{N} X \in D^{\perp}$. Then for $X, Y \in D$ we get

$g\left(A_{N} X, Y\right)=0$.

Again by using (13) we have

$g(h(X, Y), N)=g\left(A_{N} X, Y\right)=0$.

i.e., $h(X, Y)=0 \forall X, Y \in D$, which implies that $M$ is $D$-totally geodesic.

This completes the proof.

In the same way we prove our next proposition.

Proposition 5 Let $M$ be a contact CR-submanifold of an $(\varepsilon)$-paracontact Sasakian manifold $\tilde{M}$.

Then $M$ is $D^{\perp}$-totally geodesic if and only if $A_{N} X \in D$ for each $X \in D^{\perp}$, where $N$ is normal vector field to $M$.

Proof. The proof follows immediately from proposition (4) . prove,

Concerning the integrability of horizontal distribution $D$ and vertical distribution $D^{\perp}$ on $M$, we

Theorem 6 Let $M$ be a contact CR-submanifold of an $(\varepsilon)$-paracontact Sasakian manifold. Assuming $M$ to be $\xi$-horizontal, the distribution $D$ is integrable iff

$$
h(X, \phi Y)=h(\phi X, Y)
$$

$\forall X, Y \in D$ and if $M$ is $\xi$-vertical then the distribution $D^{\perp}$ is integrable iff

$$
A_{\phi X} Y-A_{\phi Y} X=\varepsilon\left[\eta(X) \phi^{2} Y-\eta(Y) \phi^{2} X\right]
$$

$\forall X, Y \in D^{\perp}$.

Proof. Assuming $M$ to be $\xi$-horizontal we have from (21)

$\forall X, Y \in D$.

$$
h(X, \phi Y)=\phi Q \nabla_{X} Y+C h(X, Y)
$$

Since $[X, Y] \in D$, we have $D$ is integrable iff

$h(X, \phi Y)=h(\phi X, Y)$.

Again using the equation (21) we have

$\nabla_{X}^{\perp} \phi Y=\phi Q \nabla_{X} Y+C h(X, Y)$

$\forall X, Y \in D^{\perp}$. Then from (9) and (16) we have

$\tilde{\nabla}_{X} \phi Y=-g(\phi X, \phi Y) \xi-\varepsilon \eta(Y) \phi^{2} X+\phi P \nabla_{X} Y+\phi Q \nabla_{X} Y+B h(X, Y)$

$+C h(X, Y)$

Again using Weingarten formula we have 


$$
\tilde{\nabla}_{X} \phi Y=-A_{\phi Y} X+\nabla_{X}^{\perp} \phi Y .
$$

From (33) and (34) we have

$$
\phi P \nabla_{X} Y=g(\phi X, \phi Y) \xi+\varepsilon \eta(Y) \phi^{2} X-A_{\phi Y} X-B h(X, Y) .
$$

Interchanging $X$ and $Y$ we have

$$
\phi P \nabla_{Y} X=g(\phi Y, \phi X) \xi+\varepsilon \eta(X) \phi^{2} Y-A_{\phi X} Y-B h(Y, X) .
$$

Substracting (36) from (35) we obtain

$$
\phi P[X, Y]=-A_{\phi Y} X+A_{\phi X} Y+\varepsilon \eta(Y) \phi^{2} X-\varepsilon \eta(X) \phi^{2} Y .
$$

Since $M$ is $\xi$-vertical, therefore $[X, Y] \in D^{\perp}$ iff

$$
A_{\phi X} Y-A_{\phi Y} X=\varepsilon\left[\eta(X) \phi^{2} Y-\eta(Y) \phi^{2} X\right] .
$$

Hence the proof is complete.

Corollary 7 Let $M$ be a $\xi$-horizontal CR-submanifold of an $(\varepsilon)$-paracontact Sasakian manifold $\tilde{M}$. Then the horizontal distribution $D$ is integrable iff

$$
g(h(X, P Y)-h(Y, P X), Q Z)=0
$$

for any $X, Y \in D$ and $Z \in D^{\perp}$.

Proof. The proof follows immediately from above theorem.

Now we shall give the definition of $D$-umbilic(resp. $D^{\perp}$ umbilic) contact CR-submanifold $M$ of an ( $\varepsilon$ ) -paracontact Sasakian manifold $\tilde{M}$.

Definition 8 A contact CR-submanifold $M$ of an $(\varepsilon)$-paracontact Sasakian manifold is said to be $D$ -umbilic (resp. $D^{\perp}$-umbilic) if $h(X, Y)=g(X, Y) L$ holds for all $X, Y \in D \quad\left(\right.$ resp. $X, Y \in D^{\perp}$ ); where $L$ being some normal vector field.

Definition (8) helps us to state proposition (9).

Proposition 9 Suppose $M$ is a $D$-umbilic (resp. $D^{\perp}$-umbilic) contact CR-submanifold of an $(\varepsilon)$ paracontact Sasakian manifold $\tilde{M}$. If $M$ is $\xi$-horizontal (resp. $\xi$-vertical) then $M$ is $D$-totally geodesic (resp. $D^{\perp}$-totally geodesic).

Proof. If $M$ be a $D$-umbilic $\xi$-horizontal contact CR-submanifold, then from definition (8)

$h(X, Y)=g(X, Y) L, \forall \quad X, Y \in D$

where $L$ is the normal vector field on $M$ and by assuming $X=Y=\xi$, we have

$h(\xi, \xi)=0$.

This implies that $g(\xi, \xi) L=0$ and therefore $L=0$ and consequently we get $h(X, Y)=0$, which proves that $M$ is $D$-totally geodesic. Similarly, it can be easily shown that if $M$ is $D^{\perp}$-umbilic $\xi$ - 
vertical contact CR-submanifold then it is $D^{\perp}$-totally geodesic.

\section{Mixed totally geodesic contact CR-submanifold of an (epsilon)-paracontact Sasakian manifold}

In this section we give the definition of mixed totally geodesic and foliate contact CR-submanifold of an $(\varepsilon)$-paracontact Sasakian manifold and study some results from these definitions.

Definition 10 A contact $C R$-submanifold $M$ of an $(\varepsilon)$-paracontact Sasakian manifold $\tilde{M}$ is said to be mixed totaly geodesic if $h(X, Y)=0 \forall X \in D$ and $Y \in D^{\perp}$ [1][2].

From definition (10) we can easily prove this lemma.

Lemma 11 Let $M$ be a contact CR-submanifold of an $(\varepsilon)$-paracontact Sasakian manifold. Then $M$ is mixed totally geodesic iff

$$
A_{N} X \in D
$$

$\forall X \in D$, and $\forall$ normal vector field $N$.

$$
A_{N} X \in D^{\perp}
$$

$\forall X \in D^{\perp}$ and normal vector field $N$.

Proof. The proof can be easily done by using definition (10) .

Using lemma (11) we obtain the following theorem.

Theorem 12 If $M$ is a mixed totally geodesic contact CR-submanifold of an $(\varepsilon)$-paracontact Sasakian manifold, then

$$
\begin{aligned}
& A_{\phi N} X=\phi A_{N} X, \\
& \nabla_{X}^{\perp} \phi N=\phi \nabla_{X}^{\perp} N,
\end{aligned}
$$

$\forall \quad X \in D$ and normal vector field $N$.

Proof. Using lemma (4.2), Gauss and Weingarten formulae (16), (17) respectively and after some brief calculations we can derive equations (42) and (43).

From [4] we have,

Definition 13 A contact CR-submanifold $M$ of an $(\varepsilon)$-paracontact Sasakian manifold $\tilde{M}$ is called foliate contact CR-submanifold $\tilde{M}$ if $D$ is involute. If $M$ is a foliate $\xi$-horizontal contact $C R$-submanifold, we know

$$
h(\phi X, \phi Y)=h\left(\phi^{2} X, Y\right)=h(X, Y) .
$$

In view of the above definition we give another proposition. 
Proposition 14 If $M$ is a foliate $\xi$-horizontal mixed totally geodesic contact CR-submanifold $M$ of an $(\varepsilon)$ - paracontact Sasakian manifold, then

$$
\phi A_{N} X=A_{N} \phi X
$$

$\forall X \in D$ and normal vector field $N$.

Proof. From (13) and (29) we compute

$$
g(h(X, \phi Y), N)=g\left(\phi A_{N} X, Y\right)
$$

i.e.

$$
g(h(\phi X, Y), N)=g\left(A_{N} \phi X, Y\right)
$$

From (46) and (47) we obtain

$$
\phi A_{N} X=A_{N} \phi X \text {. }
$$

Hence the proof follows.

\section{Geometry of leaves on CR-submanifolds of an (epsilon)-paracontact Sasakian manifold}

In this section we have obtained results on the immersions of leaves of distributions in $M$. For the leaves of involute distributions we shall obtain necessary and sufficient conditions in order that their immersions in $M$ be totally geodesic.

Proposition 15 Let $M$ be a $\xi$ horizontal CR-submanifold of an $\mathcal{E}$-paracontact Sasakian manifold $\tilde{M}$. Then the distribution $D$ is integrable and the leaf of $D$ is totally geodesic in $M$ iff

$$
g\left(h(D, D), \phi D^{\perp}\right)=0
$$

Proof. Let $X, Y \in D$ and $Z \in D^{\perp}$. If the distribution $D$ is integrable and its leaf is totally geodesic in $M$, then $\nabla_{X} \phi Y \in D$.

Using (9), (11), (18) to (20) we can calculate

$$
\begin{aligned}
& \left(\tilde{\nabla}_{X} P\right) Y=A_{Q Y} X+B h(X, Y)-g(X, Y) \xi-\varepsilon \eta(Y) P X \\
& \left(\tilde{\nabla}_{X} Q\right) Y=C h(X, Y)-h(X, P Y)-\varepsilon \eta(Y) Q X .(51)
\end{aligned}
$$

Again using (17) and (18) we obtain

$$
\begin{aligned}
& P \nabla_{X} Y=-A_{Q Y} X-B h(X, Y)-g(X, Y) \xi-\varepsilon \eta(Y) P X \\
& Q \nabla_{X} Y=\nabla_{X}^{\perp} Q Y-h(X, P Y)-\varepsilon \eta(Y) Q X
\end{aligned}
$$

For $X, Y \in D$ and $Z \in D^{\perp}$ using (52) we get

$$
\begin{aligned}
& 0=g\left(\nabla_{X} \phi Y, Z\right)=-g\left(\nabla_{X} Z, \phi Y\right)=g\left(P \nabla_{X} Z, Y\right) \\
& =-g\left(A_{F Z} X, Y\right)-g(B h(X, Z), Y)=g(h(X, Y), \phi Z)
\end{aligned}
$$

from which we obtain the required condition.

Conversely, if the given condition holds then the distribution $D$ is integrable by corollary (7). Moreover we get from (9),

$$
0=g(h(X, \phi Y), \phi Z)=g\left(\tilde{\nabla}_{X} \phi Y, \phi Z\right)=g\left(\phi \tilde{\nabla}_{X} Y, \phi Z\right)=g\left(\nabla_{X} Y, Z\right)
$$


Thus $\nabla_{X} Y \in D$ for any $X, Y \in D$ and leaf of $D$ is totally geodesic in $M$. Hence the proof is done.

Proposition 16 Let $M$ be a CR-submanifold of an $(\varepsilon)$-paracontact Sasakian manifold $\tilde{M}$. Then the leaf $M^{\perp}$ of $D^{\perp}$ is totally geodesic in $M$ iff

$$
g(h(Y, W), Q Z)-\varepsilon \eta(Y) g(W, Z)=0
$$

for any $Y \in D$ and $W, Z \in D^{\perp}$.

Proof. From (52) we have on putting $X=W \in D^{\perp}$ and $Y=Z$ we obtain $P \nabla_{W} Z=-A_{Q Z} W-B h(W, Z)-g(W, Z) \xi-\varepsilon \eta(Z) W$.

Taking inner product with $Y \in D$ and using (13) we calculate $g\left(\nabla_{W} Z, P Y\right)=g(h(Y, W), Q Z)-\varepsilon \eta(Y) g(W, Z)$.

Our assertion follows immediately from above equation.

Corollary 17 Let $M$ be a $\xi$-vertical CR-submanifold of an $(\varepsilon)$ - paracontact Sasakian manifold $\tilde{M}$. Then the leaf of $M^{\perp}$ of $D^{\perp}$ is totally geodesic in $M$ iff

$$
g\left(h(D, D), \phi D^{\perp}\right)=0
$$

\section{Contact CR-product of an (epsilon)- paracontact Sasakian manifold}

Definition 18 A submanifold $M$ of an $(\varepsilon)$-paracontact Sasakian manifold $\tilde{M}$ is called a contact CR-product if it is locally a Riemannian product of $M^{T}$ and $M^{\perp}$ where $M^{T}$ and $M^{\perp}$ are the leaves of distributions $D$ and $D^{\perp}$ resp by[13].

Theorem 19 Let $M$ be a $\xi$-horizontal CR-submanifold of an $(\varepsilon)$ - paracontact Sasakian manifold $\tilde{M}$. Then $M$ is a contact $C R$-product iff

$$
A_{\phi W} X-\varepsilon \eta(X) W=0,(56)
$$

for any $X \in D$ and $W \in D^{\perp}$

Proof. If a CR-submanifold $M$ of an $(\varepsilon)$ - paracontact Sasakian manifold $\tilde{M}$ is contact CR-product then from proposition (16) and from equation (13) we get

$$
g\left(A_{\phi W} X, Z\right)-\varepsilon \eta(X) g(W, Z)=0,
$$

for $X \in D$ and $W, Z \in D^{\perp}$. From this we get

$$
A_{\phi W} X-\varepsilon \eta(X) W \in D(58)
$$

for any $X \in D, W \in D^{\perp}$.

As $D$ is totally geodesic in $M$, we get for $Y \in D$

$$
\begin{aligned}
& g\left(A_{\phi W} X-\varepsilon \eta(X) W, Y\right)=g(h(X, Y), \phi W) \quad=-g(\phi h(X, Y), W) \\
& =-g\left(\phi \tilde{\nabla}_{X} Y-\phi \nabla_{X} Y, W\right)=-g\left(\phi \tilde{\nabla}_{X} Y, W\right)=g\left(\nabla_{X} \phi Y, W\right)=0,
\end{aligned}
$$

for any $X, Y \in D$ and $W \in D^{\perp}$.This implies that 


$$
A_{\phi W} X-\varepsilon \eta(X) W \in D^{\perp}
$$

From (56) we have

$$
g(h(X, Z)-\varepsilon \eta(X) \phi Z, \phi W)=0,
$$

for any $X \in D$ and $W, Z \in D^{\perp}$, implies that the leaf $M^{\perp}$ of $D^{\perp}$ is totally geodesic in $M$. Again suppose $M^{\perp}$ be the leaf of $D$. Then from (9) and (57) we get

$$
\begin{aligned}
& g\left(\nabla_{X} Y, Z\right)=g\left(\tilde{\nabla}_{X} Y, Z\right)=g\left(\phi \tilde{\nabla}_{X} Y, \phi Z\right)=g\left(\tilde{\nabla}_{X} \phi Y, \phi Z\right), \\
& g(h(X, \phi Y), \phi Z)=g\left(A_{\phi Z} X, \phi Y\right)=\varepsilon \eta(X) g(\phi Y, Z)=0,
\end{aligned}
$$

for any $X, Y \in D$ and $Z \in D^{\perp}$ i.e. the leaf $M^{\perp}$ of $D^{\perp}$ is totally geodesic in $M$. Thus the submanifold $M$ is a contact CR-product.

Proposition 20 Let $M$ be $\xi$-horizontal contact CR-product of an $(\varepsilon)$ - paracontact Sasakian manifold $\tilde{M}$. Then for unit vector $X \in D$ with $\eta(X)=0$ and $Z \in D^{\perp}$ we obtain

- $g\left(h\left(\nabla_{X} \phi X, Z\right), \phi Z\right)=-\varepsilon^{2}$

- $g\left(h\left(\nabla_{\phi X} X, Z\right), \phi Z\right)=\varepsilon^{2}$

- $g\left(h\left(\phi X, \nabla_{X} Z\right), \phi Z\right)=0$

- $g\left(h\left(X, \nabla_{\phi X} Z\right), \phi Z\right)=0$

Proof. As $M$ is $\xi$-horizontal contact CR-product of an $(\varepsilon)$ - paracontact Sasakian manifold $\tilde{M}$ so the leaf $M^{\perp}$ of $D^{\perp}$ is totally geodesic in $M$. Therefore on using (10) and (60) we calculate

$$
\begin{aligned}
& g\left(h\left(\nabla_{X} \phi X, Z\right), \phi Z\right)=\varepsilon \eta\left(\nabla_{X} \phi X\right) g(Z, Z)=\varepsilon g\left(\nabla_{X} \phi X, \xi\right) \\
& =-\varepsilon g\left(\nabla_{X} \xi, \phi X\right)=-\varepsilon g(\varepsilon \phi X, \phi X)=-\varepsilon^{2} g(\phi X, \phi X), \\
& =-\varepsilon^{2} . \quad \text { since } X \text { is a unit vector }
\end{aligned}
$$

Hence we have obtained the first proof. In the same way we can obtain the rest three equations.

\section{Example}

Example 21 Let $R^{3}$ be a 3 -dimensional Euclidean space with rectangular co-ordinates $(x, y, z)$. In $R^{3}$ we define

$$
\begin{aligned}
& \eta=d z, \xi=\frac{\partial}{\partial z}(61) \\
& \phi\left(\frac{\partial}{\partial x}\right)=\frac{\partial}{\partial y}, \phi\left(\frac{\partial}{\partial y}\right)=\frac{\partial}{\partial x}, \phi\left(\frac{\partial}{\partial z}\right)=0
\end{aligned}
$$

The metric $g_{1}$ and $g_{2}$ are defined as follows:

$$
g_{1}=(d x)^{2}+(d y)^{2}-(d z)^{2}
$$

and

$$
g_{2}=-(d x)^{2}-(d y)^{2}+(d z)^{2} .
$$


Then it can be easily seen that the set $\left(\phi, \xi, \eta, g_{1}\right)$ is a timelike Lorentzian almost paracontact structure while the set $\left(\phi, \xi, \eta, g_{2}\right)$ is a spacelike $(\varepsilon)$-almost paracontact metric structure in $R^{3}$. We also note that index $\left(g_{1}\right)=1$ and index $\left(g_{2}\right)=2$. All the results in this paper can be verified from this example.

\section{References}

[1] A.Bejancu, CR-submanifolds of a Kahler manifold I, Proc. Amer. Math. Soc. 89 (1978) 135-142.

[2] A.Bejancu, CR-submanifolds of a Kahler manifold II,Trans.Amer. Math. Soc. 250 (1979) 333-345

[3] A.Bejancu and K.L.Duggal, Real hypersurfaces of indefinite Kaehler manifolds, Int.J.Math.Math.Sci.16(1993), no.3, 545-556.

[4] Arindam Bhattacharyya and Bandana Das, Contact CR-Submanifolds of an Indefinite Trans-Sasakian Manifold, Int. J. Contemp. Maths. Scinces, Vol. 6, 2011, no. 26, 1271-1282.

[5] B.O’Neill, Semi-Riemannian Geometry with application to relativity, Academic Press, 1983.

[6] D.E.Blair, Riemannian Geometry of contact and cosymplectic manifolds, Progren in Mathematics, 203. Birkhouser Boston, Inc. place city Boston, MA, 2002

[7] I.Sato, On a structure similar to the almost contact structure, Tensor(N.S.) 30(3), 219-224, 1976.

[8] J.K.Beem and P.E..Ehrlich, Global Lorentzian Geometry, Marcel Dekker, New York, 1981.

[9] K.L.Duggal, Spacetime manifolds and contact structures, International journal of Mathematics and Mathematical Sciences, 13, 545-554, 1990.

[10] K.L.Duggal and B.Sahin, Lightlike submanifolds of Indefinite Sasakian manifolds, International Journal of Mathematics and Mathematical Sciences, Hindawi Publishing Corporation, (2007), 1-22.

[11] M. Kobayashi, CR-submanifolds of a Sasakian manifold, Tensor, N.S. vol. 35 (1981), 297-307.

[12] K.Matsumoto, On Lorentzian para contact manifolds, Bull. of Yamagata Univ.Nat.Sci., 12(2), 151-156, 1989.

[13] M.Hasan Shahid, Mohd. Shoeb and A.Sharfuddin, Contact CR-product of a trans-Sasakian manifold, 1994.

[14] S.Sasaki, On differential manifolds with certain structure which are closely related to almost structure, Tohoku math.J., 12(2), 459476, 1960.

[15] M.M.Tripathi, E.Kilic, S.Y.Perktas and S.Keles, Indefinite almost paracontact metric manidolds, ar Xiv:0908.2729 vi[math.DG], 2009.

[16] T.Takahashi, Sasakian manifold with Pseudo-Riemannian metric, Tohoku Math.J., 21(2), 644-653, 1969. 